\title{
2
}

\section{THE MOSQUITO}

\section{An introduction}

\section{Frances M. Hawkes and Richard J. Hopkins}

Mosquitoes are some of the most intensely studied creatures on the planet and their role in disease transmission and nuisance biting makes them worthy of such attention. There are over 3,500 species of mosquito on earth, being found everywhere except in Antarctica. Yet, from this great diversity, only a small handful can carry the pathogens that cause human disease and it is these species which have been studied most thoroughly. For the purposes of public health, this substantial body of research has helped us to understand mosquito-borne disease transmission and informed the development of mosquito and disease control methods. A fascinating spinoff of that body of research has been to reveal a complex biology, showing the mosquito's incredible and unusual behavioural, anatomical and physiological traits.

Animal behaviours are linked to the intricate displays of brightly coloured tropical birds, the long-range migrations of grazing mammals, the semaphore flashing of fireflies in a darkened landscape, together with an infinite variety of other activities and colourful patterns across the animal kingdom. Mosquitoes, like all animals, are driven by a fundamental set of needs. The behavioural organization of an individual animal species is at the core of understanding the ecology of that species. In Dutch Biologist Nikolaas Tinbergen's classic 1963 paper, "On aims and methods of ethology," he defines four categories of explanations of animal behaviour: causation, evolution, development and function (Tinbergen, 1963). In pragmatic terms, these categories can then be regarded as either "proximate" or "ultimate" causes of a behaviour. The patterns of behaviour exhibited by mosquitoes are complex and driven by sensory systems that are adapted to the environments they inhabit and the ecological niches they exploit. Their life cycle, anatomy, physiology and behaviour make these creatures both an extraordinary object of study and crucial to human culture. 


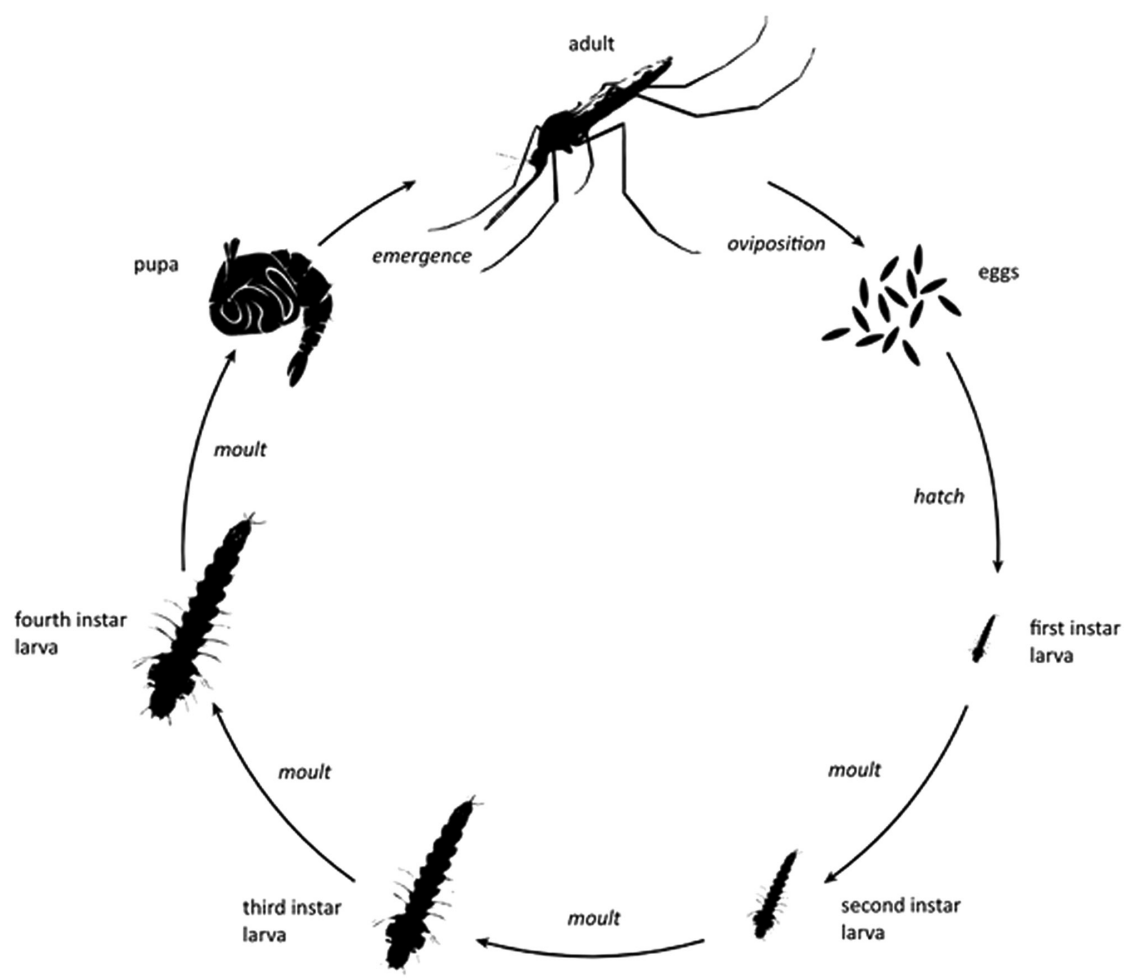

FIGURE 2.1 The mosquito life cycle. Image courtesy of Louise Malmgren/NRI.

Mosquitoes are holometabolous insects. This means that, just as caterpillars develop into butterflies, mosquitoes undergo a complete metamorphosis, hatching from eggs into larvae, then into a pupal phase (where the juvenile form liquifies and reforms into the adult body), finally hatching as fully adult flies (Figure 2.1). All mosquitoes therefore start life as an egg with a gravid female mosquito laying as many as 250 of them in each clutch. Since the mosquito's juvenile stages are aquatic, eggs must be deposited in or near water, or somewhere where water may return after flood or rainfall. The female's choice of location for laying eggs is a critical factor for determining the offspring's survival in the immature stages (eggs, larvae and pupae). Once an egg has been laid then the immature must develop in the site selected by its mother. Broadly speaking, mosquito eggs are of two types: rapid-hatch eggs which are laid directly on or adjacent to the water surface, which hatch within a couple of days; and delayed-hatch eggs typically laid adjacent to water or on moist soil or vegetation some metres distant from water. Delayed-hatch eggs can survive for long periods-months or yearsbeing resistant to desiccation and undergoing extremes of temperature that may include freezing winters. Egg laying varies widely by mosquito species, from 
single eggs to small rafts of eggs cemented to each other and placed directly on the water or else laid nearby, always ready to hatch as soon as the water level rises.

A gravid female mosquito aims to hatch her eggs in a water resource that is sufficiently rich in nutrients and long-lasting to allow the larvae to grow, develop and produce pupae from which adult mosquitoes can successfully emerge. Her selection of a laying site is dependent upon various chemical, visual, olfactory and tactile cues that influence the behaviour of the female before an egg is laid (Ignell and Hill, 2020). For some Anopheles mosquitoes, water vapour itself is attractive to gravid females (Okal et al., 2013), while tall riparian vegetation has been found to be a deterrent to laying eggs (Low et al., 2016). Whatever the egg-laying strategy of the species, the female's search for a location to lay her eggs may last several days. Mosquito flight is generally favoured by warm humid conditions, and if the wind is too strong then mosquitoes will not attempt to fly. Whilst generally associated with flights relatively close to the ground, some gravid females have been captured hundreds of metres in the air with little being known about these long-range movements.

Dictated by the availability of aquatic habitats, mosquitoes are found in a wide range of environments from the tropics to the Arctic circle. Their larvae can be found wriggling in vast marshlands, flood plains and wherever else water collects continuously or periodically, such as in small tree holes or human-made containers, which have become ideal reproductive niches for many species. Even the water-filled leaf axils of such plants as bromeliads can be mosquito larvae habitat. After hatching, the young mosquito larva harvests nutrients from the water. Larvae are essentially detritivores, filter feeding on decomposing organic matter, bacteria and algae for several weeks, spending much of their time at the water's surface to take in air, much like a snorkeler. As they build food reserves necessary to tide them through the intense process of metamorphosis, larvae can be aggressively predated on by fish, amphibians and other aquatic invertebrates. Moreover, once emerged as adults, mosquitoes transfer huge volumes of biomass into the terrestrial food web, in turn contributing to the diets of insectivorous mammals, birds and other invertebrates. So fundamental are mosquitoes in the food chain that when their numbers were controlled in the Camargue region of southern France, breeding success of the house martin, Delichon urbicum, was reduced by some 25\% compared to untreated areas (Poulin et al., 2010).

After progressing through four larval stages, or instars, the mosquito is ready to pupate. Pupae physically resemble so many commas swimming in the water, and though they do not feed, they are highly mobile and respond to the slightest threat by tumbling down through the water to escape potential predation. When the mosquito pupa is fully developed, it will rise to the surface of the water one last time. Here the adult mosquito is ready to emerge, and so straightens its body, splits open its exoskeleton and emerges upright into the air (Figure 2.2)

After resting briefly on the surface of the water, the adult mosquito must take a short shaky flight to find a refuge, typically in surrounding vegetation, where it rests to allow its newly pumped-up wings to dry and properly harden. 


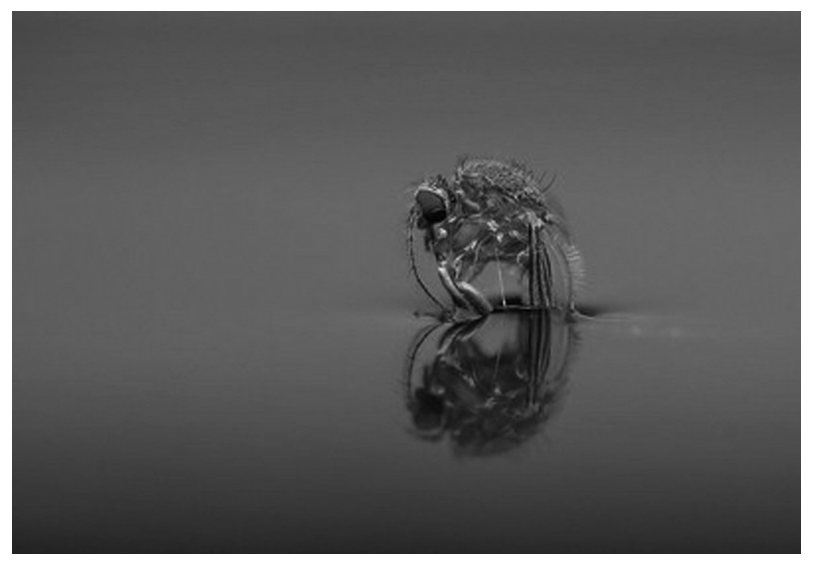

FIGURE 2.2 An emerging male Culex pipiens. Photo by Anders Lindstrom/SVA.

As a consequence of the wide range of their larval habitats, there is a correspondingly large variation in emergence patterns of different mosquito species. Whilst the number of mosquitoes that are able to grow in and emerge from the water in a tin can will always be small, the numbers emerging from larger bodies of water can be massive and dramatic. This is most apparent for species such as the inland floodwater mosquito, Aedes vexans, a highly cosmopolitan mosquito found in many countries with a range covering every continent except Antarctica and South America. Although capable of transmitting a range of human pathogens, Aedes vexans is best known for its role as a nuisance mosquito, hence its binomial name: the Latin vexare means to torment or harass. This mosquito does not lay its eggs directly in the water but in the moist soil above the waterline. After a period of drying, these eggs can survive for years waiting for the water level to rise and be sufficiently warm. If the water is too cold or clear, the eggs will not hatch. When suitable waters do come to inundate meadows or river flood plains, Aedes vexans eggs can hatch across vast areas; hundreds of larvae can be found in a litre of floodwater, equating to over 100 million larvae per hectare (Becker et al., 2010), the subsequent emergence of adult mosquitoes reaching biblical proportions. Such population levels of this, and other floodwater species, can become so extreme as to deter all normal human activities. Vexāre indeed.

Once emerged as adults, both male and female mosquitoes feed extensively on sources of plant-based sugars, such as those found in nectar and fruit juices. Only the adult female mosquito seeks a blood meal, which has the requisite nutrients to support egg development. When the female does feed on blood it does not necessarily select humans as the source of this blood, and indeed humans are rarely a mosquito's main blood source. Other mammals, birds or reptiles will also satisfy the palate of some mosquito species. It is when the mosquito selects a 
human for its blood meal that the insect earns its reputation as an annoyance and harbinger of disease.

It might also be noted that while the majority of mosquito species follow this general dietary pattern, there is a notable exception in the genus Toxorhynchites. This group includes the world's largest mosquito, Toxorhynchites speciosus, whose wingspan may be four times larger than most other species. The idea of a giant mosquito may seem somewhat terrifying; however, the 90 or so species in this genus are particularly noteworthy because the adults do not take any blood meals at all and feed exclusively on plant sugars. This is on account of the predatory nature of the larvae, which kill live prey-including other mosquito larvae-in order to acquire sufficient reserves of protein for egg development when adult. So aggressive are the predatory (and even cannibalistic) appetites of Toxorhynchites amboinensis larvae that this species has been cultivated and distributed as an effective form of mosquito biocontrol, especially for the control of species of mosquitoes that typically breed on shipping containers and which are associated with the transmission of dengue and Zika virus (Collins and Blackwell, 2000).

\section{The secret life of a mosquito}

Whilst for most people, mosquitoes are defined by an incessant whining that disturbs their sleep at night or by an intense swollen itch on their ankle, there is far more to mosquito biology than their interactions with humans. These are but brief moments in their admittedly rather short lives - in the tropics most individuals will seldom survive longer than a month as adults. Throughout the course of their adult life, mosquitoes must perform a series of complex searches for resources at different times and in different places across the landscape. They interact not only with their blood-meal host, but with plants that are also sources of food, with a mate, and with places and structures for resting and laying their eggs. The resources that each individual insect searches for vary in their spatial and temporal availability and are sometimes to be found in widely differing environments. The behaviour of a searching adult mosquito is driven by external cues in the form of chemical and visual signals that the insect must process to guide its searches.

The organs that the mosquito uses to inform the searches are all remarkable in their own way. Stimuli that are visual, chemical and aural all play important roles at some point in the life of the adult mosquito. The compound eyes of night-active mosquitoes are amongst the most sensitive to low light levels in the animal kingdom and the structure of the nocturnal mosquito eye is uniquely adapted to maximize this sensitivity. Such eye sensitivity enables visually guided flight in light conditions equivalent to moonless, starless nights, although such sensitivity comes at the expense of visual resolution, with the world appearing as a heavily pixelated image of light and dark patches. The mosquito antenna is another remarkable organ. It can detect odours that distinguish potential hosts, sugar meal sources and egg-laying (oviposition) sites. This antenna not only 
has humidity and thermal receptors, it is also endowed with one of the most sensitive sound detectors in the insect kingdom, the Johnston's organ. Described by and named after Christopher Johnston (1855), these are the most complex mechanosensitive organs yet found in insects. This doughnut-shaped organ at the base of the antenna detects minute vibrations from sound waves. The Johnston's organ's extreme sensitivity to sound allows a flying mosquito to detect the tones produced by the wingbeats of other flying mosquitoes and distinguish these from tones produced by its own wings. Fascinatingly, conspecific male and female mosquitoes not only detect the sound of each other's wingbeats, they also adjust their own wingbeat frequency to match that of their potential partner during flight, producing a harmonious duet in a prelude to mating (Gibson and Russell, 2006).

Often, all the resources that a mosquito needs can be found in a relatively small area, with conventional wisdom being that mosquitoes, on the whole, travel relatively short distances, generally less than a few hundred metres, with exceptions of up to several tens of kilometres being documented. With the high habitat diversity and host availability found in some environments, there may be little need to travel far. However, research carried out in Africa's Sahel has demonstrated that malaria mosquitoes fly hundreds of metres up, where they may be carried on the wind for distances of around 300 kilometres in a single night (Huestis et al., 2019). The majority of these insects were found to be blood-fed females, so that their travel over long distances may have significant repercussions for transmitting such diseases as malaria and for the ecological aspects of their searching for egg-laying sites during prolonged periods of drought.

In any case, flying between resources and in search of shelter requires a great deal of energy and adult mosquitoes need to feed in order to gather the energy that flight requires. Whilst people generally think of mosquitoes as exclusively blood-feeding, sugar feeding is a cornerstone of adult mosquito life. Newly emerged mosquitoes cannot survive for long without taking in a sugar meal and it is the consumption of sugar that facilitates females in their search for blood. Although female adult mosquitoes in all but a few species require a blood meal to produce eggs, both male and females feed on nectar and other sources of plant sugars to provide energy. Given the choice, the majority of female mosquitoes will consume a sugar meal before they take blood, with floral nectar being by far the most important of the sugar sources for mosquitoes. Although the phrase "sugar feeding" is commonly used, it is perhaps a misnomer for the process of mosquitoes feeding on plants, since mosquitoes gain greater value from a plant's other nutrients than simple carbohydrates. "Phytophagy" may therefore be the more appropriate way to describe mosquito feeding (Peach and Gries, 2020). It has even been further demonstrated that compounds present in nectar can differentially affect the development of malaria parasites within the mosquito to such an extent that their feeding on certain plants can suppress the malaria cycle in the insect phase (Hien et al., 2016). 
Since floral meals are the most significant contributor to mosquito phytophagy, then how do mosquitoes find this key food source? In general, it is common amongst phytophagous insects that their attraction to the plant is governed by a gestalt of signals available to guide the insect to the correct plant. It is almost certainly the case for mosquitoes seeking nectar to fuel their flights that their search is based on a range of signals coming from flowers. The visual cues associated with flowers and insects have been extensively explored for day-flying pollinators, such as bees, but less is known about floral visitation by nectar-foraging mosquitoes. Broadly speaking, nectar-foraging mosquitoes often visit flowers that are white or pale yellow to the human eye. For nocturnal species of mosquito, this can be linked to the fact that many flowers that make nectar available at night are often pale flowers, which have a strong contrast against the dark landscape, thereby offering stronger visual cues for the pollinators. In addition to visual cues, floral odours, metabolic heat and the nocturnal respiration of carbon dioxide are all associated with floral location by mosquitoes (Peach et al., 2019). Floral feeding by mosquitoes has been relatively understudied compared to other aspects of mosquito biology. It can be concluded that mosquitoes do feed on extrafloral nectaries and on fruit juices, and that mosquitoes will utilize a broader range of plant material than just flowers when flowers are scarce.

Such nectar-feeding habits mean that mosquitoes may have a role in pollination. Mosquitoes appear able to pick up small clusters of pollen during nectar feeding, thereby facilitating plant pollination, even if there are very few documented cases of obligate pollination by mosquitoes. The blunt-leaved orchid, Platanthera obtusata, which can be found in the bogs, swamps and wooded fens of northern North America, is one such mosquito-pollinated plant, with Aedes communis attracted to the orchid's chemical compounds (Lahondère et al., 2020).

While locating plant-based sugars is one occupation of male mosquitoes, their other chief activity is mate-seeking. The growing importance of research about male mate-seeking stems from an interest in mating disruption or using modified males to spread characteristics which disrupt a mosquito's ability to act as a vector of disease (Takken et al., 2006). Like most insect species, male adult mosquitoes typically emerge slightly ahead of females by a day or so, requiring that extra time to become sexually mature. When females emerge, they are often ready to mate almost immediately. It is common for many mosquito species to have mating "swarms" formed predominantly by the males, usually around dusk. These swarms often form close to visible structures or other conspicuous landmarks, although the exact mechanisms that guide the positioning of these mating swarms are poorly understood. Most female mosquitoes will mate just once during their lifetime, storing the sperm to fertilize all subsequent eggs they produce. Alongside the genetic material that allows sexual reproduction to take place, various proteins are also transferred from the male to the female during mating. This transmitted chemical concoction triggers changes in female behaviour, switching from mate-seeking to searching for blood to nourish her eggs. 
Commonplace misconceptions regarding the seemingly indomitable ability of mosquitoes to locate and bite us-and us specifically, rather than anybody else-arise from an awareness that our body odour or something of our scent is somehow detectable and traceable by mosquitoes. Olfaction is indeed the critical mechanism that females use to locate a suitable blood meal. The mosquito's primary scent-sensing organs are the antennae. These paired appendages are covered with hundreds of tiny hairs called sensilla, each capable of detecting airborne molecules, including various chemical odours emanating from animal skin (Sutcliffe, 1994). Breath also releases important telltale chemicals and mosquitoes carry a pair of sensory palpi next to the antennae crucial for detecting those, too.

Like other blood-sucking insects, adult female mosquitoes are highly attracted to carbon dioxide. Produced during respiration by every vertebrate animal and exhaled in the breath, carbon dioxide is an extremely reliable indicator of the presence of living animals and, thus, potential blood meals. Of course, carbon dioxide is also a natural component of the atmosphere, but malaria mosquitoes can detect changes in concentration of as little as $0.01 \%$. Such minute changes in concentration are sufficient to trigger flight in mosquitoes at rest (Healy and Copland, 1995). Trails of the molecule can also be identified by mosquitoes from some distance, with Anopheles melas able to detect plumes of carbon dioxide at 18 metres (Gillies and Wilkes, 1969). This first detection of carbon dioxide initiates a complex sequence of behaviours that ultimately lead the insect to its prey.

For some species of mosquito, carbon dioxide appears to be all that they require to locate the source of a blood meal; the stronger the signal, the better. In the case of Culex tarsalis, the greater the volume of carbon dioxide released, the more mosquitoes are attracted to the source (Reeves, 1953; Allan, Bernier and Kline, 2006). For Anopheles species that can carry malaria, their attraction to carbon dioxide can have important implications for the spread of mosquitoborne disease. For example, women in the later stages of pregnancy exhale about $21 \%$ more carbon dioxide per breath, meaning that, other factors remaining equal, the number of Anopheles gambiae attracted to pregnant women can double (Lindsay et al., 2000). Since such women are at greater risk of complications from malaria, this particular aspect of mosquito behaviour can produce dangerous consequences to those most vulnerable.

While the carbon dioxide in the air is a generic clue that a host may be nearby, it provides no definitive information about which kind of animal is producing the gas. For opportunist species, such as the Caribbean tree hole mosquito, Aedes mediovittatus, any blood-carrying animal is targeted, be it sheep, rat, pig, horse, cow, goat, cat, dog, chicken or human (Barrera et al., 2012). Other mosquitoes are even more catholic in their diet, with Culex erraticus, for example, also feeding on reptiles, amphibians, as well as birds, large-hooved mammals and humans (Clements, 1999).

But several medically important mosquito species are extremely specific in their preferred host. Indeed, a key factor in making Anopheles gambiae a highly 
efficient malaria vector is its faithful choice of humans for a blood meal, a trait known as anthropophily. Once infected with human malaria parasites after the first feeding, a female mosquito can feed several more times, potentially transmitting parasites to a new human host each time (a cow, to consider a different vertebrate, cannot become infected with human malaria and so suffers no ill-effects if fed upon by mosquitoes carrying human malaria; from the human point of view, every blood meal taken from non-human vertebrates is one less chance to spread infection). Anopheles gambiae evolved its extreme specialization to humans through ongoing association with agricultural communities, which provided it with reliable sources of food and niches for resting and oviposition (Besansky et al., 2004). How, then, can this species that is so discerning in its host, distinguish between all animal sources of carbon dioxide and pinpoint a human? And is every person's scent equally appetizing to a hungry mosquito?

An animal's emanations of sweat, breath and bacteria are composed of a complicated array of volatile compounds. Around 350 different chemicals have been identified in human skin odours alone (Bernier et al., 2000). Anopheles gambiae's sensitive antennae detect those chemicals that are tied most closely to humans alone. Chemical analysis and direct recording of electrical signals from live Anopheles gambiae antennae revealed the identity of these compounds (Cork and Park, 1996). Yet even if a mosquito can detect an odour compound, it does not always respond to it, making behavioural research into their responses to chemical stimuli a delicate operation, with results that may vary according to an odour's concentration, volume or the presence of other odours at the same time. Key, behaviour-influencing compounds include carboxylic acids, lactic acid and ammonia (related to sweat production and its incubation, respectively), and octenol, which is more abundant in cattle, for example, than humans and may therefore aid Anopheles gambiae in discriminating between them. All of these odours stem from communities of bacteria that live harmlessly on our skin but vary from person to person, and it is these individual differences that affect attractiveness to mosquitoes, regardless of the gender or age of the person (Verhulst et al., 2011); thus, certain unfortunate people are genuinely more attractive to malaria mosquitoes than others, simply by virtue of their unique skin microbiota (Qiu et al., 2006). Moreover, recent evidence suggests that an infection of Plasmodium parasites enhances one's production of volatile chemicals attractive to malaria mosquitoes (Robinson et al., 2018). In this way, Plasmodium seems to be luring mosquitoes in for a blood meal that will serve to propagate the plasmodium.

How valid are claims that consuming garlic or spicy foods, or vitamin B supplements, might disguise a person's odour fingerprint, and make it less appealing to mosquitoes? There is limited evidence that a person's diet can alter his or her attractiveness to a mosquito-although the results of a study about beer consumption offer some intriguing news: controlling for individual variation and baseline attractiveness, researchers found that those drinking a litre of beer caused more malaria mosquitoes to fly towards them than those drinking a litre 
of water (Lefèvre et al., 2010). Researchers speculate that ingesting alcohol causes changes in breath and volatile odours that are more attractive to Anopheles gambiae-with implications for beverage choice when needing to confront this particular mosquito.

Beyond the dangers presented by a handful of disease vectors and nuisance biters, most other mosquito species will rarely, if ever, bite a person. Much less is known of the details of life histories and ecological interactions of these other species, as they have not been deemed so worthy of research. However, some investigations are beginning to reveal the stunning complexity in other mosquito-host interactions.

Cold-blooded hosts, such as toads, frogs, salamanders, lizards and even mudskippers (amphibious fish), are important blood sources for many mosquitoes in the genera Mimomyia, Uranotaenia and Deinocerites. Since body heat has been shown to be an important signal for other mosquitoes locating hosts over short distances, these colder hosts present a challenge. It turns out that several mosquito species, including Japanese Uranotaenia yaeyamana, American palefooted Uranotaenia lowii and European Uranotaenia unguiculata, are apparently sensitive to the sounds of their hosts, since recordings of certain frog calls have been shown to attract these mosquitoes (Borkent and Belton, 2006; Tamashiro et al., 2011; Camp et al., 2018).

While the idea of mosquitoes feeding on frogs and toads may seem peculiar, there are very few groups of animals off the menu to at least one or two species of mosquito. Researchers have recently identified the mystery host animals of Uranotaenia sapphirina, a mosquito from eastern North America characterized by attractive stripes of iridescent blue scales, tracing it to various annelid worms and leeches (Reeves et al., 2018). Even fish blood has been extracted and identified from the gut of engorged Aedes baisasi mosquitoes (Tamashiro et al., 2011). DNA sequencing of blood meals has been used to verify that these mosquitoes also feed on various species of eel, goby, mudskipper, rockskipper (or blenny) and triggerfish common to mangrove lagoons and rocky reefs (Miyake et al., 2019). Many of these fish are amphibious or air-breathing and some eels will wriggle out of the water and across muddy ground, while others inadvertently expose their upperparts to air when feeding in shallow waters. Aedes baisasi is able to exploit these brief moments of vulnerability.

Whatever a mosquito's choice of host animal, the insect must undertake the risky business of landing on and puncturing the host's skin. The mouthparts of all insects are derived from common structures, with adaptations to suit their particular diets, be they nectar, grain or, in the case of mosquitoes, blood. A mosquito's long and slender mouthparts, collectively known as a proboscis, are supremely adapted to the task of blood feeding and give the mosquito a reputation as a flying syringe-yet the underlying anatomy is far more sophisticated than a simple needle. Once the female has landed on a suitable host, she begins to probe, repeatedly driving her mouthparts into the host. Using a pair of bladelike mandibles, which in other insects may grasp or slice food, the mosquito 
pins the host skin firmly in place. A second pair of serrated maxillae proceed to saw through the surface of the skin. These appendages are so sharp that the host is often quite unaware of their action. Once the skin is pierced, the mosquito inserts two hollow tubular structures through the skin to search for a blood vessel from which to draw the vital fluid. The first tube, called the labrum, moves freely, bending and curving as it probes the tissue until it detects a suitable capillary (Choumet et al., 2012). When a vessel is located, the labrum pierces it and begins drawing up the host's protein-rich blood. Meanwhile, the second tubular structure, called the hypopharynx, injects the mosquito's saliva into the surrounding tissue. This saliva contains over 100 proteins that keep the blood flowing while slowing the immune and defensive responses of the host (Vogt et al., 2018). The proteins include also anesthetics that numb the area surrounding the bite, anti-inflammatories to maintain blood pressure, vasodilators to keep blood vessels wide and anti-clotting agents to keep the blood flowing near the feeding site.

It is the host's inflammatory immune response to mosquito saliva that results in the painful, itchy welts associated with the insect's bite. Yet, the impressive array of chemicals within mosquito saliva may also include compounds of significant value in developing the next generation of pharmaceutical medicines. Researchers have found that anophelin, the salivary protein produced by Anopheles gambiae as an anti-coagulant, can be modified for helping to dissolve human blood clots, thereby opening up new possibilities for developing novel drugs that can prevent stroke and deep vein thrombosis (Watson et al., 2018).

A mosquito's reliance on blood feeding is the characteristic that allows mosquitoes to transmit pathogens from infected to healthy hosts. Pathogens are taken up by mosquitoes incidentally when the latter are imbibing a blood meal. These viruses and parasites, which accumulate in the mosquito's salivary glands, are then injected into new hosts along with saliva during the probing phase. However, this is not the full story, since only a small fraction of the 3,500 mosquito species can transmit human pathogens, mostly limited to those of just three genera: Anopheles, Aedes and Culex. Only mosquitoes of the genus Anopheles can transmit human malaria parasites, and only three species of over 500 described anophelines are responsible for the majority of malaria transmission. It is worth remembering that most species of mosquitoes rarely, or never, bite humans, having specialized instead to take blood meals from other mammalian, avian, reptilian or amphibian hosts.

Moreover, the simple exchange of blood and saliva is insufficient for making a mosquito into a vector, at least not instantaneously. Most mosquito-borne pathogens must undergo a process of replication or development within the body of the mosquito, which can take up to 23 days for Plasmodium vivax (Thomas et al., 2018), but can be as quick as two days in the case of dengue viruses, depending on environmental conditions (Chan and Johansson, 2012). Should the insect feed on another host before this process is complete, she will not yet be able to pass on viable infective agents. Yet proof that the mosquito can successfully imbibe blood 
from infected hosts, and then inject pathogen-laden saliva into uninfected hosts, is shown by the nearly 700 million cases of mosquito-borne illnesses occurring each year (World Mosquito Program, 2020).

It would be a mistake to assume that the presence of the pathogen is unproblematic to the mosquito, for even the immune system of the vector will attempt to tackle the invading pathogen (Rodrigues et al., 2010). A female mosquito consuming a meal from a malaria-infected human can ingest thousands of gametocytes (the stage of malaria parasites found in infected human blood). By digesting these gametocytes and exposing them to other toxic processes, her immune system can reduce their numbers from thousands to the tens (Smith et al., 2014). Moreover, white blood cells belonging to the host animal and ingested by the mosquito during feeding will also continue to target the malaria parasite for hours after entering the mosquito gut (Lensen et al., 1997). But even this two-pronged attack can fail to halt the malaria parasite's growth. Despite these gametocyte elimination processes, a small number may still survive to form oocysts (the parasite's next developmental stage) on the outside of the mosquito's gut lining. When mature, these burst open, each releasing thousands of sporozoites, which migrate to the mosquito's salivary glands ready to infect the next suitable host. The successful development of just one oocyst is, therefore, all it takes for the mosquito to become infectious.

Vectors, too, can be stricken by the effects of the pathogens which infect them following a blood meal. Dengue virus, for instance, detrimentally affects the fecundity and fitness of its principal vector, Aedes aegypti. Dengue-infected mosquitoes lay fewer or no eggs, with adult longevity being halved (Sylvestre et al., 2013). Malaria parasites can also upset the normal reproductive processes in anophelines. By promoting cell death in the lining of the insect midgut, the rodent malaria, Plasmodium yoelii, is found to cause Anopheles stephensi to reabsorb ovarian follicles, essentially destroying the next clutch of eggs the female would have produced (Hopwood et al., 2001).

In other considerations, certain parasites have been shown to manipulate their hosts for enhancing their own chances of survival. Malaria parasites have highly complex associations with their mosquito and vertebrate hosts, and it has been suggested that these single-celled protozoa may also influence mosquito physiology and behaviour to increase the probability of their survival and transmission. The introduction of a malaria parasite into a mosquito vector undoubtedly changes the mosquito, although whether such changes are truly an adaptive manipulation or simply a side effect of infection can be difficult to determine (Hurd, 2003). Nonetheless, several studies suggest that parasites produce physiological and behavioural changes in mosquitoes that favour their onward transmission.

It has been determined that wild Anopheles gambiae mosquitoes infected with the most deadly human malaria parasite, Plasmodium falciparum, take blood meals from more than one person in a single night, whereas uninfected mosquitoes are more likely to feed on a single individual. Such multiple feeding behaviour 
increases the number of contacts between the infected mosquito vector and human hosts, thereby increasing the transmission potential of the parasite (Koella et al., 1998). Laboratory studies also emphasize the complexity of the vector-host relationship. Research has shown that Anopheles gambiae mosquitoes infected with Plasmodium berghei (a strain of rodent malaria) were willing to probe a mouse's skin more often than uninfected mosquitoes (Choumet et al., 2012). Although the source of this behaviour is unknown, it may nonetheless confer advantage to the parasite by increasing the likelihood that a mosquito successfully feeds on blood and passes the parasites to the next host.

Ultimately, we realize that the unflattering reputation of mosquitoes may be well-earned. Their visceral parasitic strategy for acquiring nutrition can certainly appear more gruesome, and less honourable, than other predators going about their lives in the animal kingdom. That they stalk us and frequently attack in stealth, often under the cover of darkness, and target specific individuals may be perceived at some level as both devious and personal. That their feeding aggravates us, disturbs our rest, creates an itch that can stay for days and may even infect us with debilitating and potentially deadly disease elevates mosquitoes to the level of the positively dastardly. It may therefore seem unsurprising for those of us involved in the study of this insect to be asked, "What is the point of a mosquito?" Although scientists aim to avoid teleological explanations for natural phenomena, some of the less commonly studied aspects of mosquito biology already demonstrate biotic interactions that are much more complicated than those of a simple blood-sucking pest and carrier of disease. Although we are just beginning to uncover some of their more cryptic behaviours, much work remains to be done. Thousands of mosquito species have evolved marvelous and intricate biological adaptations for generating diverse behavioural and ecological traits that are still unknown to science. The activities of mosquitoes in the ecosystem are as sophisticated and specialized as that of any other creature, and indeed more complex than many. The study of mosquito biology may reveal biochemical, anatomical and behavioural secrets that may not only enrich our understanding of nature but also become a source of bioinspiration in future sciences and technologies, from the design of pain-free microneedles (Gurera et al., 2018) to algorithms for flying drones (Nakata et al., 2020). Critically, the overwhelming majority of insects that fall within the Culicidae do not pose a threat to human health or comfort, so caution must be exercised when discussing "mosquitoes", generically, as carriers of disease. For the mosquitoes that are vectors of disease, it is the pathogens and parasites they harbour which cause us morbidity and mortality, and in some cases not without cost to the infected mosquito itself. This is key. While malaria cannot persist without mosquitoes, mosquitoes can persist without malaria, or dengue, or Zika. When driven to distraction by the whining of a mosquito or the itch from their bite, many will not realize that "that wretched mosquito" is but one of myriad species each occupying a unique niche in the environment. Appreciating these subtleties in how we frame debates about 
"mosquito eradication" can inform a more nuanced discussion, where these key differences call for differences in our response.

\section{Bibliography}

Allan, S.A., Bernier, U.R. and Kline, D.L. 2006. Laboratory evaluation of avian odors for mosquito (diptera: culicidae) attraction. Journal of Medical Entomology, 43(2), pp. 225-231.

Barrera, R. et al. 2012. Vertebrate hosts of aedes aegypti and aedes mediovittatus (diptera: culicidae) in rural Puerto Rico. Journal of Medical Entomology, 49(4), pp. 917921. Available at: http://ovidsp.ovid.com/ovidweb.cgi? $T=J S \& P A G E=$ reference $\& D$ $=$ emed10\&NEWS $=$ N\&AN $=22897052$.

Becker, N. et al. 2010. Mosquitoes and Their Control, Second Edition. Heidelberg: Springer. doi:10.1007/978-3-540-92874-4.

Bernier, U.R. et al. 2000. Analysis of human skin emanations by gas chromatography/ mass spectrometry. 2. Identification of volatile compounds that are candidate attractants for the yellow fever mosquito (Aedes aegypti). Analytical Chemistry, 72(4), pp. 747-756. doi:10.1021/ac990963k.

Besansky, N.J., Hill, C.A. and Costantini, C. 2004. No accounting for taste: Host preference in malaria vectors. Trends in Parasitology, 20(6), pp. 249-251. doi:10.1016/j. pt.2004.03.007.

Borkent, A. and Belton, P. 2006. Attraction of female Uranotaenia lowii (diptera: culicidae) to frog calls in costa attraction of female Uranotaenia lowii (diptera: culicidae) to frog calls in Costa Rica. Canadian Entomologist, 138, pp. 91-94. doi:10.4039/N04-113.

Camp, J.V. et al. 2018. Uranotaenia unguiculata Edwards, 1913 are attracted to sound, feed on amphibians, and are infected with multiple viruses. Parasites and Vectors, 11(1), pp. 1-10. doi:10.1186/s13071-018-3030-2.

Chan, M. and Johansson, M.A. 2012. The incubation periods of dengue viruses. PLoS ONE, 7(11), pp. 1-7. doi:10.1371/journal.pone.0050972.

Choumet, V. et al. 2012. Visualizing non infectious and infectious anopheles gambiae blood feedings in naive and saliva-immunized mice. PLoS ONE, 7(12). doi:10.1371/ journal.pone.0050464.

Clements, A.N. 1999. The Biology of Mosquitoes Volume 2. Oxfordshire: CABI Publishing.

Collins, L.E. and Blackwell, A. 2000. The biology of toxorhynchites mosquitoes and their potential as biocontrol agents. Biocontrol News and Information, 21(4), pp. 105N-116N.

Cork, A. and Park, K.C. 1996. Identification of electrophysiologically-active compounds for the malaria mosquito, anopheles gambiae, in human sweat extracts. Medical and Veterinary Entomology, 10(3), pp. 269-276.

Gibson, G. and Russell, I. 2006. Flying in tune: Sexual recognition in mosquitoes. Current Biology, 16(13), pp. 1311-1316. doi:10.1016/j.cub.2006.05.053.

Gillies, M.T. and Wilkes, T.J. 1969. A comparison of the range of attraction of animal baits and of carbon dioxide for some West African mosquitoes. Bulletin of Entomological Research, 59(3), pp. 441-456. Available at: https://www.cambridge.org/core/jour nals/bulletin-of-entomological-research/article/comparison-of-the-range-of-attra ction-of-animal-baits-and-of-carbon-dioxide-for-some-west-african-mosquitoes/ DF0C87E573834F02844F8C07BFB7DFF5.

Gurera, D., Bhushan, B. and Kumar, N. 2018. Lessons from mosquitoes' painless piercing. Journal of the Mechanical Behavior of Biomedical Materials. Elsevier Ltd, 84(November 2017), pp. 178-187. doi:10.1016/j.jmbbm.2018.05.025. 
Healy, T. and Copland, M. 1995. Activation of anopheles gambiae mosquitoes by carbon dioxide and human breath. Medical and Veterinary Entomology, 9(3), pp. 331-336.

Hien, D.F.d.S. et al. 2016. Plant-mediated effects on mosquito capacity to transmit human Malaria. PLoS Pathogens, 12(8), pp. 1-17. doi:10.1371/journal.ppat.1005773.

Hopwood, J.A. et al. 2001. Malaria-induced apoptosis in mosquito ovaries: A mechanism to control vector egg production. Journal of Experimental Biology, 204(16), pp. 2773-2780.

Huestis, D.L. et al. 2019. Windborne long-distance migration of malaria mosquitoes in the Sahel. Nature. Springer US, 574(7778), pp. 404-408. doi:10.1038/s41586-019-1622-4.

Hurd, H. 2003. Manipulation of medically important insect vectors by their parasites. Annual Review of Entomology, 48(1), pp. 141-161. doi:10.1146/annurev. ento.48.091801.112722.

Ignell, Rickard and Sharon Rose Hill. 2020. Malaria mosquito chemical ecology. Current Opinion in Insect Science, 40, doi:10.1016/j.cois.2020.03.008

Johnston, C. 1855. Auditory apparatus of the Culex Mosquito. Journal of Cell Science, 3, pp. 97-102.

Koella, J.C., Sorensen, F.L. and Anderson, R.A. 1998. The malaria parasite, Plasmodium falciparum, increases the frequency of multiple feeding of its mosquito vector, anopheles gambiae. Proceedings of the Royal Society B: Biological Sciences, 265(1398), pp. 763-768. doi:10.1098/rspb.1998.0358.

Lahondère, C. et al. 2020. The olfactory basis of orchid pollination by mosquitoes. Proceedings of the National Academy of Sciences of the United States of America, 117(1), pp. 708-716. doi:10.1073/pnas.1910589117.

Lefèvre, T. et al. 2010. Beer consumption increases human attractiveness to malaria mosquitoes. PLoS ONE, 5(3), pp. 1-8. doi:10.1371/journal.pone.0009546.

Lensen, A.H.W. et al. 1997. Leukocytes in a plasmodium falciparum-infected blood meal reduce transmission of malaria to anopheles mosquitoes. Infection and Immunity, 65(9), pp. 3834-3837. doi:10.1128/iai.65.9.3834-3837.1997.

Lindsay, S. et al. 2000. Effect of pregnancy on exposure to malaria mosquitoes for personal use only. Not to be reproduced without permission of. The Lancet, 355, p. 2000.

Low, M. et al. 2016. The importance of accounting for larval detectability in mosquito habitat-association studies. Malaria Journal. BioMed Central, 15(1), pp. 1-9. doi:10.1186/s12936-016-1308-4.

Miyake, T. et al. 2019. Bloodmeal host identification with inferences to feeding habits of a fish-fed mosquito, Aedes baisasi. Scientific Reports, 9(1), pp. 1-8. doi:10.1038/ s41598-019-40509-6.

Nakata, T. et al. 2020. Aerodynamic imaging by mosquitoes inspires a surface detector for autonomous flying vehicles. Science, 368(6491), pp. 634-637. doi:10.1126/science. aaz9634.

Okal, M.N. et al. 2013. Water vapour is a pre-oviposition attractant for the malaria vector anopheles gambiae sensu stricto. Malaria Journal, 12(1), pp. 1-8. doi:10.1186/1475-2875-12-365.

Peach, D.A.H. et al. 2019. Multimodal floral cues guide mosquitoes to tansy inflorescences. Scientific Reports, 9(1), pp. 1-10. doi:10.1038/s41598-019-39748-4.

Peach, D.A.H. and Gries, G. 2020. Mosquito phytophagy - sources exploited, ecological function, and evolutionary transition to haematophagy. Entomologia Experimentalis et Applicata, 168(2), pp. 120-136. doi:10.1111/eea.12852.

Poulin, B., Lefebvre, G. and Paz, L. 2010. Red flag for green spray: Adverse trophic effects of Bti on breeding birds. Journal of Applied Ecology, 47(4), pp. 884-889. doi:10.1111/j.1365-2664.2010.01821.x. 
Qiu, Y.T. et al. 2006. Interindividual variation in the attractiveness of human odours to the malaria mosquito anopheles gambiae s.s. Medical and Veterinary Entomology, 20(3), pp. 280-287. doi:10.1111/j.1365-2915.2006.00627.x.

Reeves, L.E. et al. 2018. Identification of Uranotaenia sapphirina as a specialist of annelids broadens known mosquito host use patterns. Communications Biology. Springer US, 1(1), pp. 1-8. doi:10.1038/s42003-018-0096-5.

Reeves, W.C. 1953. Quantitative field studies on a carbon dioxide chemotropism of mosquitoes. American Journal of Tropical Medicine, 2(2), pp. 325-331. Available at: http: //www.ajtmh.org/content/journals/10.4269/ajtmh.1953.2.325.

Robinson, A. et al. 2018. Plasmodium-associated changes in human odor attract mosquitoes. Proceedings of the National Academy of Sciences of the United States of America, 115(18), pp. E4209-E4218. doi:10.1073/pnas.1721610115.

Rodrigues, J. et al. 2010. Hemocyte differentiation mediates innate immune memory in anopheles gambiae mosquitoes. Science, 239(5997), pp. 1353-1355. doi:10.1038/ jid.2014.371.

Smith, R.C., Vega-Rodríguez, J. and Jacobs-Lorena, M. 2014. The plasmodium bottleneck: Malaria parasite losses in the mosquito vector. Memorias do Instituto Oswaldo Cruz, 109(5), pp. 644-661. doi:10.1590/0074-0276130597.

Sutcliffe, J.F. 1994. Sensory bases of attractancy: Morphology of mosquito olfactory sensilla-- a review. Journal of the American Mosquito Control Association, 10(2 Pt 2), pp. 309-315.

Sylvestre, G., Gandini, M. and Maciel-de-Freitas, R. 2013. Age-dependent effects of oral infection with dengue virus on aedes aegypti (diptera: culicidae) feeding behavior, survival, oviposition success and fecundity. PLoS ONE, 8(3), pp. 1-8. doi:10.1371/ journal.pone.0059933.

Takken, W. et al. 2006. Mosquito mating behaviour. In Knols, B. and Louis, C. (eds.) Bridging Laboratory and Field Research for Genetic Control of Disease Vectors. Springer Netherlands, pp. 183-188. doi:10.1007/1-4020-3799-6_17.

Tamashiro, M. et al. 2011. Bloodmeal identification and feeding habits of mosquitoes (diptera: culicidae) collected at five islands in the Ryukyu archipelago, Japan. Medical Entomology and Zoology, 62(1), pp. 53-70. doi:10.7601/mez.62.53.

Thomas, S. et al. 2018. Microclimate variables of the ambient environment deliver the actual estimates of the extrinsic incubation period of plasmodium vivax and plasmodium falciparum: A study from a malaria-endemic urban setting, Chennai in India. Malaria Journal. BioMed Central, 17(1), pp. 1-17. doi:10.1186/s12936-018-2342-1.

Tinbergen, N. 1963. On aims and methods in ethology. Zeitschrift fur Tierpsychologie, 20, pp. $410-433$.

Verhulst, N.O. et al. 2011. Composition of human skin microbiota affects attractiveness to malaria mosquitoes. PLoS ONE, 6(12). doi:10.1371/journal.pone.0028991.

Vogt, M. et al. 2018. Mosquito saliva alone has profound effects on the human immune system. PLOS Neglected Tropical Diseases, 12(5), pp. 1-27. doi:10.1371/journal.

Watson, E.E. et al. 2018. Mosquito-derived anophelin sulfoproteins are potent antithrombotics. ACS Central Science, 4(4), pp. 468-476. doi:10.1021/ acscentsci.7b00612.

World Mosquito Program. 2020. Mosquito-Borne Diseases. Available at: https://www.wor ldmosquitoprogram.org/en/learn/mosquito-borne-diseases. 